\title{
TRACES OF BMO-SOBOLEV SPACES
}

\author{
ROBERT S. STRICHARTZ
}

\begin{abstract}
The trace operator $R F(x)=F(x, 0)$ where $F(x, t)$ is a function of $x \in \mathbf{R}^{n}$ and $t \in \mathbf{R}^{1}$ maps $I_{\alpha}(B M O)$, the $B M O$-Sobolev space of Riesz potentials of order $\alpha$ of functions of bounded mean oscillation on $\mathrm{R}^{n+1}$, onto the homogeneous Besov space $\Lambda_{\alpha}^{0}(\infty, \infty)$ on $\mathbf{R}^{n}$, for $\alpha>0$. A right inverse is given by the extension operator $E f(x, t)=\mathscr{F}^{-1}\left(e^{-t^{2}|\xi|^{2}} \hat{f}(\xi)\right)$.
\end{abstract}

1. Introduction. The space $I_{\alpha}(B M O)$ of Riesz potentials of order $\alpha$ of functions of bounded mean oscillation has been studied in [4], [8], and [9] as a substitute for the $L^{p}$-Sobolev spaces when $p=\infty$. One point left open in [8] is the characterization of the traces of functions in $I_{\alpha}(B M O)$ for $\alpha>0$. Since $I_{\alpha}(B M O) \subseteq \Lambda_{\alpha}^{0}(\infty, \infty)$ (this was proved in [8] and was essentially known earlier) and the trace of functions in $\Lambda_{\alpha}^{0}(\infty, \infty)$ must obviously also be in $\Lambda_{\alpha}^{0}(\infty, \infty)$ for $\alpha>0$, the trace operator $R F(x)=F(x, 0)$ maps $I_{\alpha}(B M O)$ into $\Lambda_{\alpha}^{0}(\infty, \infty)$. In this paper we will prove that the mapping is onto by showing that the extension operator

$$
\begin{aligned}
E f(x, t) & =\mathscr{F}^{-1}\left(e^{-t^{2}|\xi|^{2}} f(\xi)\right) \\
& =(4 \pi)^{-n / 2} t^{-n} \int f(x-y) e^{-|y|^{2} / 4 t^{2}} d y
\end{aligned}
$$

maps $\Lambda_{\alpha}^{0}(\infty, \infty)$ into $I_{\alpha}(B M O)$ for all $\alpha \geqslant 0$. This is an exact analogue of the well-known theorem of Gagliardo [3] and Stein [6] that $R$ maps $I_{\alpha}\left(L^{p}\right)$ onto $\Lambda_{\alpha-1 / p}^{0}(p, p)$ for $1<p<\infty$ and $\alpha>1 / p$, with the same extension operator. The trace theorem can be routinely transplanted to the context of compact manifolds and submanifolds; in particular the $L^{p}$ estimates for elliptic boundary value problems [1] are valid for $B M O-S o b o l e v$ spaces on the manifold and Besov spaces on the boundary.

2. Preliminaries. A general reference for all unexplained notation is Stein [7]. A locally integrable (real or complex valued) function defined on $\mathbf{R}^{n}$ is said to be of bounded mean oscillation if the mean oscillation of $f$ on any cube $Q$

$$
M O(f, Q)=\frac{1}{|Q|} \int_{Q}|f(x)-M(f, Q)| d x
$$

is un formly bounded, where $M(f, Q)$ denotes the mean of $f$ on $Q$,

$$
M(f, Q)=\frac{1}{|Q|} \int_{Q} f(x) d x
$$

Received by the editors July 18, 1980 and, in revised form, December 19, 1980.

1980 Mathematics Subject Classification. Primary 46E35.

Key words and phrases. Bounded mean oscillation, Sobolev space, Besov space, trace theorem.

1 Research supported in part by NSF grant MCS-8002771. 
and $|Q|$ denotes the Lebesgue measure of $Q$. The Banach space $B M O$ consists of functions of bounded mean oscillation modulo constants equipped with the norm $\|f\|_{B M O}=\sup _{Q} M O(f, Q)$.

The Riesz potentials are defined on the space of tempered distributions modulo polynomials by $I_{\alpha} f=\mathscr{F}^{-1}\left(|\xi|^{-\alpha} \hat{f}(\xi)\right)$, where $\alpha \in \mathbf{R}$, so we may define the $B M O$ Sobolev spaces $I_{\alpha}(B M O)$ as the image of $B M O$ under $I_{\alpha}$.

We will be interested only in the case $\alpha \geqslant 0$ when $I_{\alpha}(B M O)$ consists of locally integrable functions modulo polynomials (with a little care one can define $I_{\alpha}(B M O)$ as a space of functions modulo polynomials of degree $\left.<\alpha\right)$. In the important special case when $\alpha$ is an integer we can describe $I_{\alpha}(B M O)$ more succinctly as the space of functions whose derivatives of order exactly $\alpha$ are $B M O$.

The homogeneous Besov space $\Lambda_{\alpha}^{0}(\infty, \infty)$ is for noninteger $\alpha>0$ simply the usual Hölder class of order $\alpha$, with Zygmund's modification of using higher difference for integer $\alpha$. Thus for $0<\alpha<1, f \in \Lambda_{\alpha}^{0}(\infty, \infty)$ if and only if $\mid f(x+y)$ $-\left.f(x)|<M| y\right|^{\alpha}$ with the least $M$ the $\Lambda_{\alpha}^{0}(\infty, \infty)$-norm, identifying functions that differ by constants, while for $\alpha=1$ the condition is $\mid f(x+2 y)-2 f(x+y)+$ $f(x)|<M| y \mid$. There are myriad equivalent characterizations of these spaces; our preference is for one due to Peetre [5] that treats all values of $\alpha$ simultaneously: $f \in \Lambda_{\alpha}^{0}(\infty, \infty)$ if and only if $\left\|\sigma_{s} * f\right\|_{\infty}<M s^{\alpha}$ for all $s>0$ where $\sigma_{s}(x)=$ $s^{-n} \sigma(x / s)$ are the dilations of a fixed test function $\sigma$ (note $\hat{\sigma}_{s}(\xi)=\hat{\sigma}(s \xi)$ ) which satisfies the conditions

(1) $\hat{\sigma} \in \mathscr{D}$ with support in an annular ring, say $\frac{1}{4}<|\xi|<4$.

(2) $\hat{\sigma}=1$ in a smaller annular ring, say $\frac{1}{2}<|\xi|<2$.

(3) $\hat{\sigma}$ is radial and nonnegative.

The least $M$ is equivalent to the $\Lambda_{\alpha}^{0}(\infty, \infty)$-norm. The exact choice of $\sigma$ does not change the class $\Lambda_{\alpha}^{0}(\infty, \infty)$, and conditions (2) and (3) can be considerably weakened. We note in passing that $\int_{0}^{\infty} \hat{\sigma}(s \xi)^{2}(d s / s)$ is a positive radial function homogeneous of degree zero, hence a nonzero constant for $\xi \neq 0$, from which we can conclude

$$
f=c \int_{0}^{\infty} \sigma_{s} * \sigma_{s} * f \frac{d s}{s}
$$

for certain constant $c$ provided we identify functions modulo polynomials. This is perhaps the key identity in the entire theory of Besov spaces.

Now let $x \in \mathbf{R}^{n}$ and $t \in \mathbf{R}^{1}$. We will use lower case letters such as $f$ to denote functions of $x$ and upper case letters such as $F$ to denote functions of $x$ and $t$. The trace operator $R F(x)=F(x, 0)$ is not always well defined for locally integrable $F$. However if $F \in I_{\alpha}(B M O)$ for $\alpha>0$ then $F \in \Lambda_{\alpha}^{0}(\infty, \infty)$ (see [8, Theorem 3.4]) and so is continuous. From the Hölder-Zygmund description of $\Lambda_{\alpha}^{0}(\infty, \infty)$ it follows that $R$ maps $\Lambda_{\alpha}^{0}(\infty, \infty)$ of $\mathbf{R}^{n}$ to $\Lambda_{\alpha}^{0}(\infty, \infty)$ of $\mathbf{R}^{n-1}$ for $\alpha>0$.

The extension operator

$$
E f(x, t)=\mathscr{F}^{-1}\left(e^{-t^{2}|\xi|^{2}} \hat{f}(\xi)\right)
$$

is well defined for any tempered distribution $f$, and obviously is right-inverse to $R$, $R E f=f$. 
THEOREM. $E$ is a bounded operator from $\Lambda_{\alpha}^{0}(\infty, \infty)$ to $I_{\alpha}(B M O)$ for all $\alpha>0$.

3. Proof of Theorem. We will prove the theorem in the special case when $\alpha=k$, an integer; the result in general then follows by interpolation since both scales of spaces are preserved by the complex method of interpolation (see [2] for Besov spaces and [8] for $B M O$-Sobolev spaces). Thus suppose $f \in \Lambda_{k}^{0}(\infty, \infty)$, so

$$
\left\|\sigma_{s} * f\right\|_{\infty}<M s^{k}
$$

where $M=\|f\|_{\Lambda_{k}^{0}(\infty, \infty)}$. To prove the theorem for $\alpha=k$ we need to show that $E f \in I_{k}(B M O)$, or equivalently $(\partial / \partial x)^{\beta}(\partial / \partial t)^{j} E f \in B M O$ for all integers $j$ and multi-indices $\beta$ with $j+|\beta|=k$. Writing $(\partial / \partial x)^{\beta}(\partial / \partial t)^{j} E f=G$ we need to show

$$
M O(G, Q)<c M
$$

for every cube $Q$ in $\mathbf{R}^{n+1}$ with sides parallel to the axes, where $M$ is the constant in (3.1) and $c$ is independent of $f$ (the value of $c$ may vary from equation to equation). Any such cube $Q$ is of the form $Q_{r} \times I_{r}$ where $Q_{r}$ is a cube in $\mathbf{R}^{n}$ of side length $r$ and $I_{r}$ is an interval in $\mathbf{R}^{1}$ of length $r$.

Now from the identity (2.1) we have

$$
G=\int_{0}^{\infty}\left(\frac{\partial}{\partial x}\right)^{\beta}\left(\frac{\partial}{\partial t}\right)^{j} E\left(\sigma_{s} * \sigma_{s} * f\right) \frac{d s}{s},
$$

and given the cube $Q$ and hence $r$ we can split $G$ into two parts $G=G_{1}+G_{2}$, where we take the integral from 0 to $r$ in (3.3) for $G_{1}$, and from $r$ to $\infty$ for $G_{2}$. To establish (3.2) we will show

$$
\frac{1}{r} \int_{I_{r}} \sup _{x}\left|G_{1}(x, t)\right| d t \leqslant c M
$$

and

$$
\frac{1}{r} \int \sup _{I_{r} x Q}\left|G_{2}(x, t)-G_{2}\left(x_{0}, t_{0}\right)\right| d t<c M
$$

where $\left(x_{0}, t_{0}\right)$ is the center of $Q$, for then

$$
\begin{aligned}
M O(G, Q) & \leqslant 2 M\left(\left|G-G_{2}\left(x_{0}, t_{0}\right)\right|, Q\right) \\
& \leqslant 2 M\left(\left|G_{1}\right|, Q\right)+2 M\left(\left|G_{2}-G_{2}\left(x_{0}, t_{0}\right)\right| Q\right)<c M .
\end{aligned}
$$

To establish (3.4) and (3.5) we need to examine the form of $G$. Since $E f=$ $\mathscr{F}^{-1}\left(e^{-t^{2}|\xi|^{2}} \hat{f}(\xi)\right)$ we have

$$
G=\left(\frac{\partial}{\partial x}\right)^{\beta}\left(\frac{\partial}{\partial t}\right)^{j} E f=\mathscr{F}^{-1}\left(p(t \xi) e^{-t^{2}|\xi|^{2}} q_{k}(\xi) \hat{f}(\xi)\right)
$$

where $p$ and $q_{k}$ are polynomials in $\mathbf{R}^{n}$ and $q_{k}$ is homogeneous of degree $k$. If we write

$$
h_{t}(x)=\mathscr{F}^{-1}\left(p(t \xi) e^{-t^{2}|\xi|^{2}} q_{k}(\xi)\right)
$$

then

$$
G_{1}=\int_{0}^{r} \sigma_{s} * h_{t} * \sigma_{s} * f \frac{d s}{s}
$$


hence

$$
\begin{aligned}
\sup _{x}\left|G_{1}(x, t)\right| & <\int_{0}^{r}\left\|\sigma_{s} * h_{t}\right\|_{1}\left\|\sigma_{s} * f\right\|_{\infty} \frac{d s}{s} \\
& <M \int_{0}^{r} s^{k-1}\left\|\sigma_{s} * h_{t}\right\|_{1} d s .
\end{aligned}
$$

LEMMA. $\left\|\sigma_{s} * h_{t}\right\|_{1}<g(t / s) e^{-c t^{2} / s^{2}} s^{-k}$ for some polynomial $g$.

Proof. We have

$$
\sigma_{s} * h_{t}=\mathscr{F}^{-1}\left(\hat{\sigma}(s \xi) p(t \xi) e^{-t^{2}|\xi|^{2}} q_{k}(\xi)\right)
$$

The $L^{1}$-norm is unchanged by the change of variable $\xi \rightarrow s^{-1} \xi$ on the Fourier transform side, so

$$
\left\|\sigma_{s} * h_{t}\right\|_{1}=s^{-k}\left\|\mathscr{F}^{-1}\left(\hat{\sigma}(\xi) p\left(t s^{-1} \xi\right) e^{-t^{2}|\xi|^{2} / s^{2}} q_{k}(\xi)\right)\right\|_{1}
$$

Thus it suffices to show

$$
\left\|\sigma * h_{t}\right\|_{1} \leqslant g(t) e^{-c t^{2}} .
$$

But this follows easily from the well-known estimate

$$
\left\|\sigma * h_{t}\right\|_{1} \leqslant c \sum_{|\beta|<n+1}\left\|\left(\frac{\partial}{\partial \xi}\right)^{\beta}\left(\sigma * h_{t}\right)^{\wedge}(\xi)\right\|_{1}
$$

since $\hat{\sigma}$ has support in an annular ring. Q.E.D.

Returning to the proof of the theorem, we apply the lemma to obtain

$$
\begin{aligned}
\frac{1}{r} \int_{I_{r}} \sup _{x}\left|G_{1}(x, t)\right| d t & \leqslant M \frac{1}{r} \int_{I_{r}} \int_{0}^{r} g(t / s) e^{-c t^{2} / s^{2}} s^{-1} d s d t \\
& \leqslant M \frac{1}{r} \int_{0}^{r} \int_{0}^{\infty} g(t / s) e^{-c t^{2} / s^{2}} d t s^{-1} d s \\
& =M \frac{1}{r} \int_{0}^{r} \int_{0}^{\infty} g(t) e^{-c t^{2}} d t d s=c M
\end{aligned}
$$

which proves (3.4).

Turning to (3.5), we first write

$$
G_{2}(x, t)-G_{2}\left(x_{0}, t_{0}\right)=\left(G_{2}(x, t)-G_{2}\left(x_{0}, t\right)\right)+\left(G_{2}\left(x_{0}, t\right)-G_{2}\left(x_{0}, t_{0}\right)\right)
$$

and estimate the two differences separately. For the first we have

$$
\begin{aligned}
& \left|\int_{r}^{\infty} \int\left(\sigma_{s} * h_{t}(x-y)-\sigma_{s} * h_{t}\left(x_{0}-y\right)\right) \sigma_{s} * f(y) d y \frac{d s}{s}\right| \\
& \leqslant M \int_{r}^{\infty} \int\left|\sigma_{s} * h_{t}(x-y)-\sigma_{s} * h_{t}\left(x_{0}-y\right)\right| d y s^{k-1} d s .
\end{aligned}
$$

But by the fundamental theorem of the calculus

$$
\begin{aligned}
\int\left|\sigma_{s} * h_{t}(x-y)-\sigma_{s} * h_{t}\left(x_{0}-y\right)\right| d y \\
\quad<\left|x-x_{0}\right| \int_{0}^{1} \int\left|\nabla_{x} \sigma_{s} * h_{t}\left(x_{0}-y+\lambda\left(x-x_{0}\right)\right)\right| d y d \lambda \\
\quad<\left|x-x_{0}\right|\left\|\nabla_{x} \sigma_{s} * h_{t}\right\|_{1} .
\end{aligned}
$$


For $x \in Q_{r}$ we have $\left|x-x_{0}\right| \leqslant c r$ so

$$
\sup _{x \in Q}\left|G_{2}(x, t)-G\left(x_{0}, t\right)\right| \leqslant M c r \int_{r}^{\infty}\left\|\nabla_{x} \sigma_{s} * h_{t}\right\|_{1} s^{k-1} d s .
$$

The second difference can be estimated, for $t \in I_{r}$, using the mean value theorem, by

$$
\begin{aligned}
\left|G_{2}\left(x_{0}, t\right)-G_{2}\left(x_{0}, t_{0}\right)\right| & \leqslant \int_{r}^{\infty}\left\|\left(t-t_{0}\right) \frac{\partial}{\partial t} \sigma_{s} * h_{t_{1}}\right\|_{1}\left\|\sigma_{s} * f\right\|_{\infty} \frac{d s}{s} \\
& \leqslant M r \int_{r}^{\infty}\left\|\frac{\partial}{\partial t} \sigma_{s} * h_{t_{1}}\right\|_{1} s^{k-1} d s
\end{aligned}
$$

where $t_{1} \in I_{r}$. But now all $x$ and $t$ first derivatives of $\sigma_{s} * h_{t}$ are of the same form with $k$ increased by 1 , so we may apply the Lemma to estimate both $\left\|\nabla_{x} \sigma_{s} * h_{t}\right\|_{1}$ and $\left\|(\partial / \partial t)\left(\sigma_{s} * h_{t_{1}}\right)\right\|_{1}$ by $c s^{-k-1}$ since $g(t) e^{-t^{2}}$ is bounded. Thus

$$
\frac{1}{r} \int \sup _{I_{r} x \in Q}\left|G_{2}(x, t)-G_{2}\left(x_{0}, t_{0}\right)\right| d t<c M \int_{I_{r}} \int_{r}^{\infty} s^{-2} d s d t=c M
$$

which establishes (3.5) and completes the proof of the theorem.

\section{REFERENCES}

1. S. Agmon, A. Douglis and L. Nirenberg, Estimates near the boundary for solutions of elliptic partial differential equations satisfying general boundary conditions. I, Comm. Pure Appl. Math. 12 (1959), 623-727.

2. A. P. Calderon, Intermediate spaces and interpolation, the complex method, Studia Math. 24 (1964), 113-190.

3. E. Gagliardo, Caratterizzazioni delle trace sulla frontiera relative ad alcune classi di funzioni in $n$ variabili, Rend. Sem. Mat. Padua 27 (1957), 284-305.

4. U. Neri, Fractional integration in the space $H^{1}$ and its dual, Studia Math. 53 (1975), 175-189.

5. J. Peetre, Sur les espaces de Besov, C. R. Acad. Sci. Paris Ser. A-B 264 (1967), A281-A283.

6. E. M. Stein, The characterization of functions arising as potentials. II, Bull. Amer. Math. Soc. 68 (1962), 577-582.

7. Singular integrals and differentiability properties of functions, Princeton Univ. Press, Princeton, N.J., 1970.

8. R. Strichartz, Bounded mean oscillation and Sobolev spaces, Indiana Univ. Math. J. 29 (1980), 539-558.

9. A. Torchinsky and R. Wheeden, On the Marcinkiewicz integral and functions of bounded mean ascillation, Indiana Univ. Math. J. 29 (1980), 479-494.

Department of Mathematics, Cornell University, Ithaca, New York 14853 